\title{
Synthesis and anticonvulsant activity of substituted thiourea derivatives
}

\author{
Ahmet Özgür Çelen¹, Bedia Kaymakçıoğlu, Salih Gümrü2, Hale Zerrin Toklu², Feyza Arıcıoğlu²
}

\begin{abstract}
Twelve new thiourea derivatives were prepared by the reacting of 4-aminophenylacetic acide with substituted isothiocyanates. Their chemical structures were proved by means of IR, 1H-NMR, mass spectroscopic and elemental analyses. These compounds were tested at dose of $50 \mathrm{mg} / \mathrm{kg}$ for their anticonvulsant activity using by pentylenetetrazole induced seizure (PTZ) and maximal electroshock seizure (MES) tests in mice. Compound $1 \mathrm{~b}$, (4-\{[(4-chlorophenyl)thiocarbamoyl] amino\} phenyl)acetic acid, was found to be more active than the other tested compounds. The compound $1 \mathrm{~b}$ reduced convulsions in all types of grades (from grade I to V), therefore it increased convulsive threshold. It also increased onset time from 1.20 to $2.58 \mathrm{sec}$. and survival \% from 50 to 95 .
\end{abstract}

KEY WORDS: Synthesis, thiourea, anticonvulsant activity.

\section{INTRODUCTION}

Thioureas are important sulphur and nitrogencontaining compounds and they are useful substances in drug research. Some thiourea derivatives possess valuable biological pharmacological activities such as, anti-HIV / antiviral (1-4), antitubercular (5-8), analgesic (9-10) and anticancer properties (11-13). In addition, urea and thioureas (14-16) have emerged as structurally novel anticonvulsant.

In the past 15 years, 13 new antiepileptic drugs (AEDs) have been introduced, some of which are advantageous in terms of pharmacokinetics, tolerability, and potential for drug interactions. These AEDs are regarded as second generation compared with older AEDs, such as phenobarbital, phenytoin, carbamazepine, ethosuximide, and valproic acid. However, the second-generation AEDs marketed so far have not been a breakthrough because, altogether, their use leads to freedom from seizures in no more than $15-20 \%$ of patients with epilepsy who are refractory to older AEDs. Therefore, despite the current availability of more than 15 drugs, about $30 \%$ of people with epilepsy have uncontrolled disease, and novel and more eff ective third-generation AEDs are needed (17).

As a part of our ongoing research program pertaining to the synthesis of series of thiourea and urea derivatives as potent anticonvulsant activi- ties $(18,19)$. Among this series, the compounds $N$ Ethyl-N'-(3,5-dimethylpyrazole-4-yl)thiourea (I) and $N$-(2-Ethoxyphenyl)-N'-(3,5-dimethylpyrazole-4-yl)

urea (II) were found to show a better anticonvulsant activity (Figure 1). In the MES test, these compounds exhibited median effective doses $\left(\mathrm{ED}_{50}\right)$ of 17.14 and $17.46 \mathrm{mg} / \mathrm{kg}$ respectively.<smiles>CCNC(=S)Nc1c(C)n[nH]c1C</smiles>

(I)

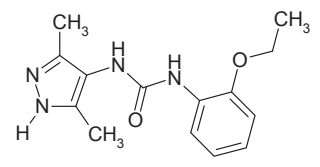

(II)
FIGURE 1. Chemical formulas of compound (I) and compound (II)

The anticonvulsant drug design was based on the presumption that for the evaluation of the anticonvulsant activity with maximal electroshock treatment (MES) at least one phenyl or similar aromatic group in close proximity to two electron donor atoms were required and that for the evaluation with pentylenetetrazole (PTZ) an alkyl group substituted close to two electron donor atoms was required (20). As a part of our continuous research, we designed compounds $\mathbf{1 a - 1 1}$ according to pharmacophoric features with the one phenyl ring as a hydrophobic aryl ring, serving as thiourea and carboxylic acide group to provide an electron donor/acceptor system (Figure
AFFILIATIONS

${ }^{1}$ Marmara Üniversitesi

Eczacılık Fakültesi,

Farmasötik Kimya Anabilim

dalı, İstanbul, Türkiye

2Marmara Üniversitesi

Eczacılık Fakültesi,

Farmakoloji Anabilim Dalı,

İstanbul, Türkiye

CORRESPONDENCE

Bedia Kaymakçıoğlu

E-mail: bkaymakcioglu@

marmara.edu.tr

Received:

17.01.2011

Accepted:

05.03.2011 
TABLE 1. Structure and physical data of thiourea derivatives 1a-Il

\begin{tabular}{|c|c|c|c|c|}
\hline Compound & $\mathrm{Ar}$ & Formula & M. p. (oC) & Yield (\%) \\
\hline $1 a$ & 4-F-C6H4- & C15H13FN2O2S & $200-201$ & 67 \\
\hline $1 b$ & 4-Cl-C6H4- & $\mathrm{C} 15 \mathrm{H} 13 \mathrm{CIN} 2 \mathrm{O} 2 \mathrm{~S}$ & $206-207$ & 48 \\
\hline $1 \mathrm{c}$ & 2,4,6-triCl-C6H2- & $\mathrm{C} 15 \mathrm{H} 11 \mathrm{Cl} 3 \mathrm{~N} 2 \mathrm{O} 2 \mathrm{~S}$ & $214-215$ & 50 \\
\hline $1 d$ & 4- $\mathrm{CH} 3-\mathrm{C} 6 \mathrm{H} 4-$ & $\mathrm{C} 16 \mathrm{H} 16 \mathrm{~N} 2 \mathrm{O} 2 \mathrm{~S}$ & $215-216$ & 56 \\
\hline $1 e$ & 4- $\mathrm{CH} 3 \mathrm{O}-\mathrm{C} 6 \mathrm{H} 4-$ & $\mathrm{C} 16 \mathrm{H} 16 \mathrm{~N} 2 \mathrm{O} 3 \mathrm{~S}$ & 193-194 & 61 \\
\hline $1 f$ & 4-CH3S-C6H4- & C16H16N2O2S2 & $206-207$ & 70 \\
\hline $1 \mathrm{~g}$ & 4-CF3-C6H4- & $\mathrm{C} 16 \mathrm{H} 13 \mathrm{~F} 3 \mathrm{~N} 2 \mathrm{O} 2 \mathrm{~S}$ & 197-198 & 45 \\
\hline $1 \mathrm{~h}$ & 4-CF3O-C6H4- & $\mathrm{C} 16 \mathrm{H} 13 \mathrm{~F} 3 \mathrm{~N} 2 \mathrm{O} 3 \mathrm{~S}$ & $210-211$ & 42 \\
\hline $1 \mathrm{i}$ & 4-NO2-C6H4- & $\mathrm{C} 15 \mathrm{H} 13 \mathrm{~N} 3 \mathrm{O} 4 \mathrm{~S}$ & $139-140$ & 63 \\
\hline $1 \mathrm{j}$ & $\mathrm{C} 6 \mathrm{H} 5-\mathrm{CH} 2-$ & $\mathrm{C} 16 \mathrm{H} 16 \mathrm{~N} 2 \mathrm{O} 2 \mathrm{~S}$ & $178-179$ & 67 \\
\hline $1 \mathrm{k}$ & $\mathrm{C} 6 \mathrm{H}_{5}-\mathrm{CH} 2-\mathrm{CH} 2-$ & $\mathrm{C} 17 \mathrm{H} 18 \mathrm{~N} 2 \mathrm{O} 2 \mathrm{~S}$ & $170-171$ & 64 \\
\hline 11 & $\mathrm{C} 6 \mathrm{H} 5-\mathrm{CO}-$ & $\mathrm{C} 16 \mathrm{H} 14 \mathrm{~N} 2 \mathrm{O} 3 \mathrm{~S}$ & $168-169$ & 55 \\
\hline
\end{tabular}

2). The other phenyl ring served as a second hydrophobic region.

$$
\text { la-1l }
$$

FIGURE 2. General chemical formula of compounds $1 \mathrm{a}-1 \mid$

The current work encompasses synthesis of a new series of thioureas by reaction of (4-aminophenyl)acetic acid with different isothiocyanates and evaluation for anticonvulsant activity using by PTZ and MES tests in mice.

\section{EXPERIMENTAL}

\subsection{Chemistry}

All chemicals and solvents were purchased from Merck, Aldrich, or Fluka. Melting points were determined with a "Schmelzpunktbestimmer" SMP II and were uncorrected. ${ }^{1} \mathrm{H}-$ NMR spectra were recorded in DMSO on a Bruker AvanceDPX-400 spectrometer in DMSO- $\mathrm{d}_{6}$ and chemical shifts were given in $\delta \mathrm{ppm}$ with tetramethylsilane. The splitting patterns of ${ }^{1} \mathrm{H}-\mathrm{NMR}$ were designed as follows: s: singlet, $\mathrm{d}$ : doublet, $\mathrm{t}$ : triplet, q: quarlet, m: multiplet. The Mass spectrometry was performed using an Agilent 1100 MSD spectrometer in the electrospray mode. All new compounds were analyzed for $C$, $\mathrm{H}, \mathrm{N}$ and the results were in an acceptable range $\left({ }^{1} \mathrm{H}-\mathrm{NMR}\right.$, mass and elemental analysis were provided by the Scientific and Technical Research Council of Turkey, TUBITAK).

General procedure for the preparation of $1 \mathrm{a}-1 \mathrm{l}$

$0.500 \mathrm{~g}$ (3.3 mmol) 4-(Aminophenyl)acetic acid is solved in acetone at $100^{\circ} \mathrm{C}$. Then, a solution of corresponding isothiocyanate $(3.3 \mathrm{mmol})$ in $5 \mathrm{~mL}$ acetone is added as three parts per 30 minutes. After 6-8 hours, reaction is finalized by TLC control. Solid material is filtered and recrystallized from acetonitrile.

(4-\{[(4-Fluorophenyl)thiocarbamoyl]amino\}phenyl)acetic acid (1a): UV $\lambda_{\text {max. }}(\mathrm{EtOH})(\mathrm{nm})(\log \mathrm{e}): 275(3,98)$. IR Spectroscopy $\left(\mathrm{u}_{\max }, \mathrm{cm}^{-1}\right): 3196(\mathrm{~N}-\mathrm{H}, \mathrm{O}-\mathrm{H}), 3005(\mathrm{C}-\mathrm{H}), 1693(\mathrm{C}=\mathrm{O}), 1236$ $(\mathrm{C}=\mathrm{S}) .{ }^{1} \mathrm{H}-\mathrm{NMR}(400 \mathrm{MHz})\left(\mathrm{DMSO}-\mathrm{d}_{6} / \mathrm{TMS}\right) \mathrm{d}(\mathrm{ppm}): 3.55$ $\left(2 \mathrm{H}, \mathrm{s},-\mathrm{CH}_{2}\right), 6.87-7.71(8 \mathrm{H}, \mathrm{m}$, aromatic protons $), 9.72(1 \mathrm{H}, \mathrm{s}$, -NH-), 9.72 (1H, s, -NH-), $12.26(1 \mathrm{H}, \mathrm{s}, \mathrm{OH})$. Anal. Calcd. for
$\mathrm{C}_{15} \mathrm{H}_{13} \mathrm{FN}_{2} \mathrm{O}_{2}$ S; C: \% 59.20; H: \% 4.31; N: \% 9.20; S: \% 10.54. Found: C: \% 57.55; H: \% 4.30; N: \% 8.74; S: \% 9.63.

(4-\{[(4-chlorophenyl)thiocarbamoyl]amino\}phenyl)acetic acid (1b) UV $\lambda_{\max }(\mathrm{EtOH})(\mathrm{nm})(\log \mathrm{e}): 278(3,51)$. IR $\left(\mathrm{u}_{\max }, \mathrm{cm}^{-1}\right)$ : 3194 (N-H, O-H), 3012 (C-H), 1689 (C=O), 1240 (C=S). ${ }^{1} \mathrm{H}-$ NMR (400 MHz) (DMSO-d 6 / TMS) d (ppm): $3.47\left(2 \mathrm{H}, \mathrm{s},-\mathrm{CH}_{2}\right)$, 7.15-7.59 (8H, m, aromatic protons), $9.68(1 \mathrm{H}, \mathrm{s},-\mathrm{NH}-), 9.79$ $(1 \mathrm{H}, \mathrm{s},-\mathrm{NH}-), 11.98(1 \mathrm{H}, \mathrm{s}, \mathrm{OH})$. Anal. Calcd. for $\mathrm{C}_{15} \mathrm{H}-$ ${ }_{13} \mathrm{ClN}_{2} \mathrm{O}_{2} \mathrm{~S}$; C: \% 56.16; H: \% 4.08; N: \% 8.73; S: \% 10.00. Found: C: \% 57.80; H: \% 4.49; N: \% 8.38; S: \% 9.34.

(4-\{[(2,4,6-trichlorophenyl)thiocarbamoyl]amino\}phenyl)acetic acid (1c): UV $\lambda_{\text {max. }}(\mathrm{EtOH})(\mathrm{nm})(\log \mathrm{e}): 259(3,63)$. IR $\left(\mathrm{u}_{\max }\right.$ $\left.\mathrm{cm}^{-1}\right)$ : 3155 (N-H, O-H), 2989 (C-H), 1693 (C=O), 1224 (C=S). ${ }^{1} \mathrm{H}-\mathrm{NMR}(400 \mathrm{MHz})\left(\mathrm{DMSO}-\mathrm{d}_{6} / \mathrm{TMS}\right) \mathrm{d}$ (ppm): 3.56 (2H, s, $\left.-\mathrm{CH}_{2}\right), 7.18-7.79(6 \mathrm{H}, \mathrm{m}$, aromatic protons $), 9.39(1 \mathrm{H}, \mathrm{s},-\mathrm{NH}-)$, $9.93(1 \mathrm{H}, \mathrm{s},-\mathrm{NH}-), 12.32(1 \mathrm{H}, \mathrm{s}, \mathrm{OH})$. Anal. Calcd. for $\mathrm{C}_{15} \mathrm{H}-$ ${ }_{11} \mathrm{Cl}_{3} \mathrm{~N}_{2} \mathrm{O}_{2} \mathrm{~S}$; C: \% 46.23; H: \% 2.85; N: \% 7.19; S: \% 8.23. Found: C: \% 46.69; H: \% 3.07; N: \% 7.15; S: \% 7.93.

(4-\{[(4-methylphenyl)thiocarbamoyl]amino\}phenyl)acetic acid (1d): UV $\lambda_{\max .}(\mathrm{EtOH})(\mathrm{nm})(\log \mathrm{e}): 278(3,55)$. IR $\left(\mathrm{u}_{\max }, \mathrm{cm}^{-1}\right)$ : 3201 (N-H, O-H), 3001 (C-H), 1695 (C=O), 1238 (C=S). ${ }^{1} \mathrm{H}-$ NMR (400 MHz) (DMSO-d 6 /TMS) d (ppm): $2.22\left(2 \mathrm{H}, \mathrm{s},-\mathrm{CH}_{2}\right)$, $3.26\left(3 \mathrm{H}, \mathrm{s},-\mathrm{CH}_{3}\right), 7.10-7.50(8 \mathrm{H}, \mathrm{m}$, aromatic protons $), 9.55$ (1H, s, -NH-), 9.74 (1H, s, -NH-), $12.31(1 \mathrm{H}, \mathrm{s}, \mathrm{OH})$. Anal. Calcd. for $\mathrm{C}_{16} \mathrm{H}_{16} \mathrm{~N}_{2} \mathrm{O}_{2} \mathrm{~S}$; C: \% 62.95; $\mathrm{H}$ : \% 5.58; N: \% 8.16; S: \% 9.34. Found: C: \% 61.21; H: \% 4.96; N: \% 8.55; S: \% 9.71.

(4-\{[(4-methoxyphenyl)thiocarbamoyl]amino\}phenyl)acetic acid (1e): $U V \lambda_{\max }(\mathrm{EtOH})(\mathrm{nm})(\log \mathrm{e}): 276(3,34)$. IR $\left(\mathrm{u}_{\max }, \mathrm{cm}^{-}\right.$ 1): 3215 (N-H, O-H), 3026 (C-H), 1689 (C=O), 1234 (C=S). ${ }^{1} \mathrm{H}-$ NMR (400 MHz) (DMSO-d 6 TMS) d (ppm): $3.49\left(2 \mathrm{H}, \mathrm{s},-\mathrm{CH}_{2}\right)$, $3.69\left(3 \mathrm{H}, \mathrm{s},-\mathrm{CH}_{3}\right), 6.90\left(2 \mathrm{H}, \mathrm{d}, \mathrm{J}=8.94 \mathrm{~Hz}, \mathrm{H} 3^{\prime}, \mathrm{H}^{\prime}\right), 7.20(2 \mathrm{H}$, d, J= $8.41 \mathrm{~Hz}, \mathrm{H} 2, \mathrm{H} 6), 7.30\left(2 \mathrm{H}, \mathrm{d}, \mathrm{J}=8.93 \mathrm{~Hz}, \mathrm{H} 2^{\prime}, \mathrm{H} 6^{\prime}\right), 7.40$ $(2 \mathrm{H}, \mathrm{d}, \mathrm{J}=8.41 \mathrm{~Hz}, \mathrm{H} 3, \mathrm{H} 5), 9.46$ (1H, s, -NH-), 9.46 (1H, s, -NH), $12.08(1 \mathrm{H}, \mathrm{s}, \mathrm{OH})$. Anal. Calcd. for $\mathrm{C}_{16} \mathrm{H}_{16} \mathrm{~N}_{2} \mathrm{O}_{3} \mathrm{~S}$; C: \% 60.74; H: \% 5.10; N: \% 8.85; S: \% 10.14. Found: C: \% 59.95; H: \% 4.90; N: \% 8.75; S: \% 9.81 .

(4-\{[(4-Methylsulfanylphenyl)carbamothioyl ]amino\}phenyl) acetic acid (1f): UV $\lambda_{\max }(\mathrm{EtOH})(\mathrm{nm})(\log \mathrm{e}): 279$ (3,12). IR ( $\left.\mathrm{u}_{\max }, \mathrm{cm}^{-1}\right)$ : 3209 (N-H, O-H), 3007 (C-H), 1695 (C=O), 1242 $(\mathrm{C}=\mathrm{S}) .{ }^{1} \mathrm{H}-\mathrm{NMR}(400 \mathrm{MHz})\left(\mathrm{DMSO}-d_{6} / \mathrm{TMS}\right) \mathrm{d}(\mathrm{ppm}): 3.35$ 
$\left(3 \mathrm{H}, \mathrm{s},-\mathrm{CH}_{3}\right), 3.54\left(2 \mathrm{H}, \mathrm{s},-\mathrm{CH}_{2}\right), 7.17-7.47(8 \mathrm{H}, \mathrm{m}$, aromatic protons), 9.75 (1H, s, -NH-), $9.75(1 \mathrm{H}, \mathrm{s},-\mathrm{NH}-), 12.31(1 \mathrm{H}, \mathrm{s}$, $\mathrm{OH})$. Anal. Calcd. for $\mathrm{C}_{16} \mathrm{H}_{16} \mathrm{~N}_{2} \mathrm{O}_{2} \mathrm{~S}_{2} ; \mathrm{C}$ : \% 57.81; $\mathrm{H}$ : \% 4.85; N: \% 8.43; S: \% 19.29. Found: C: \% 57.87; H: \% 4.75; N: \% 8.38; S: \% 19.21.

(4-\{[(4-Trifluoromethylphenyl)carbamothioyl]amino\}phenyl) acetic acid (1g): $\mathrm{UV} \lambda_{\max }(\mathrm{EtOH})(\mathrm{nm})(\log \mathrm{e}): 281(3,86)$. IR $\left(\mathrm{u}_{\max }, \mathrm{cm}^{-1}\right)$ : $3196(\mathrm{~N}-\mathrm{H}, \mathrm{O}-\mathrm{H}), 3014(\mathrm{C}-\mathrm{H}), 1683(\mathrm{C}=\mathrm{O}), 1242$ $(\mathrm{C}=\mathrm{S}) .{ }^{1} \mathrm{H}-\mathrm{NMR}(400 \mathrm{MHz})\left(\mathrm{DMSO}-d_{6} / \mathrm{TMS}\right) \mathrm{d}(\mathrm{ppm}): 3.54$ $\left(2 \mathrm{H}, \mathrm{s}, \mathrm{CH}_{2}\right), 7.20-7.80(8 \mathrm{H}, \mathrm{m}$, aromatic protons $), 9.74(1 \mathrm{H}, \mathrm{s}$, -NH-), 10.11 (1H, s, -NH-), $12.27(1 \mathrm{H}, \mathrm{s}, \mathrm{OH})$. Anal. Calcd. for $\mathrm{C}_{16} \mathrm{H}_{13} \mathrm{~F}_{3} \mathrm{~N}_{2} \mathrm{O}_{2} \mathrm{~S}$; C: \% 54.23; H: \% 3.70; N: \% 7.91; S: \% 9.05. Found: C: \% 55.02; H: \% 4.21; N: \% 7.94; S: \% 9.00.

(4-\{[(4-Trifluoromethoxyphenyl)carbamothioyl]amino\}phenyl)acetic acid (1h): $\mathrm{UV} \lambda_{\text {max. }}(\mathrm{EtOH})(\mathrm{nm})(\log \mathrm{e}): 278(3,80)$. IR $\left(\mathrm{u}_{\max }, \mathrm{cm}^{-1}\right)$ : 3201 (N-H, O-H), $3016(\mathrm{C}-\mathrm{H}), 1693$ (C=O), 1244 $(\mathrm{C}=\mathrm{S}) .{ }^{1} \mathrm{H}-\mathrm{NMR}(400 \mathrm{MHz})\left(\mathrm{DMSO}-d_{6} / \mathrm{TMS}\right) \mathrm{d}(\mathrm{ppm}): 3.54$ $\left(2 \mathrm{H}, \mathrm{s},-\mathrm{CH}_{2}\right), 7.09-7.68(8 \mathrm{H}, \mathrm{m}$, aromatic protons $), 9.73(1 \mathrm{H}, \mathrm{s}$, -NH-), $9.88(1 \mathrm{H}, \mathrm{s},-\mathrm{NH}-), 12.28(1 \mathrm{H}, \mathrm{s}, \mathrm{OH})$. Anal. Calcd. for $\mathrm{C}_{16} \mathrm{H}_{13} \mathrm{~F}_{3} \mathrm{~N}_{2} \mathrm{O}_{3} \mathrm{~S}$; C: \% 51.89; H: \% 3.54; N: \% 7.56; S: \% 8.66. Found: C: \% 53.54; H: \% 3.87; N: \% 7.61; S: \% 8.50.

(4-\{[(4-Nitrophenyl)thiocarbamoyl]amino\}phenyl)acetic acid (1i): $\mathrm{UV} \lambda_{\max }(\mathrm{EtOH})(\mathrm{nm})(\log \mathrm{e}): 242(4,11)$. IR $\left(\mathrm{u}_{\max }, \mathrm{cm}^{-1}\right)$ : 3564 (N-H, O-H), 3323 (C-H), 1683 (C=O), 1298 (C=S). ${ }^{1} \mathrm{H}-$ NMR (400 MHz) (DMSO-d 6 /TMS) d (ppm): $3.55\left(2 \mathrm{H}, \mathrm{s},-\mathrm{CH}_{2}\right)$, 7.11-8.36 (8H, m, aromatic protons), 10.26 (1H, s, -NH-), 10.39 $(1 \mathrm{H}, \mathrm{s},-\mathrm{NH}-), 12.30(1 \mathrm{H}, \mathrm{s}, \mathrm{OH})$. Anal. Calcd. for $\mathrm{C}_{15} \mathrm{H}_{13} \mathrm{~N}_{3} \mathrm{O}_{4} \mathrm{~S}$; C: \% 54.37; H: \% 3.95; N: \% 12.68; S: \% 9.68. Found: C: \% 53.76; H: \% 4.25; N: \%12.11; S: \% 8.32.

(4-[(Benzylthiocarbamoyl)amino]phenyl)acetic acid (1j): UV $\lambda_{\text {max. }}(\mathrm{EtOH})(\mathrm{nm})(\log \mathrm{e}): 258(3,70) . \mathrm{IR}\left(\mathrm{u}_{\max }, \mathrm{cm}^{-1}\right): 3252(\mathrm{~N}-\mathrm{H}$, O-H), 3057-3030 (C-H), 1687 (C=O), 1290 (C=S). ${ }^{1} \mathrm{H}-\mathrm{NMR}(400$ $\mathrm{MHz})\left(\mathrm{DMSO}-\mathrm{d}_{6} / \mathrm{TMS}\right) \mathrm{d}(\mathrm{ppm}): 3.52\left(2 \mathrm{H}, \mathrm{s},-\mathrm{CH}_{2}\right), 4.73(2 \mathrm{H}$, $\left.\mathrm{s},-\mathrm{CH}_{2}\right), 7.10-7.47(9 \mathrm{H}, \mathrm{m}$, aromatic protons $), 8.12(1 \mathrm{H}, \mathrm{s},-\mathrm{NH}-$ ), $9.56(1 \mathrm{H}, \mathrm{s},-\mathrm{NH}-), 12.30(1 \mathrm{H}, \mathrm{s}, \mathrm{OH})$. Anal. Calcd. for $\mathrm{C}_{16} \mathrm{H}_{16} \mathrm{~N}_{2} \mathrm{O}_{2} \mathrm{~S}$; C: \% 63.98; H: \% 5.37; N: \% 9.33; S: \% 10.67. Found: C: \% 63.73; H: \% 5.31; N: \% 9.20; S. \% 10.54.

\begin{tabular}{llcccc} 
TABLE 2. Anticonvulsant activity results of the compounds \\
\hline Compound & dose, $\mathbf{~ m g / k g}$ & \multicolumn{2}{c}{ PTZ test (\%) } & \multicolumn{2}{c}{ MES test (\%) } \\
& & Grade 5 & Survival & Grade 5 & Survival \\
\hline Control & 0 & 52 & 50 & 55 & 60 \\
$1 \mathrm{a}$ & 50 & 85 & 40 & 68 & 75 \\
$1 \mathrm{~b}$ & 50 & $10^{* \star *}$ & $95^{\star * *}$ & 85 & 40 \\
$1 \mathrm{c}$ & 50 & 76 & 60 & 90 & 70 \\
$1 \mathrm{~d}$ & 50 & 85 & 40 & 68 & 75 \\
$1 \mathrm{e}$ & 50 & 100 & 39 & 98 & 58 \\
$1 \mathrm{f}$ & 50 & 89 & 20 & 68 & 65 \\
$1 \mathrm{~g}$ & 50 & 61 & 38 & 80 & 60 \\
$1 \mathrm{~h}$ & 50 & 55 & 92 & 83 & 75 \\
$1 \mathrm{i}$ & 50 & 90 & 12 & 98 & 60 \\
$1 \mathrm{j}$ & 50 & 100 & 23 & 90 & 72 \\
$1 \mathrm{k}$ & 50 & 65 & 90 & 87 & 52 \\
$1 \mathrm{l}$ & 50 & 85 & 45 & 80 & 70 \\
\hline
\end{tabular}

Each group consists of 6-10 mice. Compounds were compaired to contol group and statistical significance is expressed as ${ }^{* *} p<0,001$.
(4-\{[(2-Phenylethyl)thiocarbamoyl]amino\}phenyl)acetic acid (1k): UV $\lambda_{\max .}(\mathrm{EtOH})(\mathrm{nm})(\log \mathrm{e}): 250(4,19)$. IR $\left(\mathrm{u}_{\max }, \mathrm{cm}^{-1}\right)$ : 3184 (N-H, O-H), 3024 (C-H), 1695 (C=O), 1298 (C=S). ${ }^{1} \mathrm{H}-$ NMR (400 MHz) (DMSO-d 6 /TMS) d (ppm): 2.85 (2H, t, phenethyl $\left.\mathrm{CH}_{2}\right), 3.52\left(2 \mathrm{H}, \mathrm{s}, \mathrm{CH}_{2}\right), 3.70\left(2 \mathrm{H}, \mathrm{t}\right.$, phenethyl $\left.\mathrm{CH}_{2}\right), 7.00-$ $7.50(9 \mathrm{H}, \mathrm{m}$, aromatic protons), $7.67(1 \mathrm{H}, \mathrm{s},-\mathrm{NH}-), 9.50(1 \mathrm{H}, \mathrm{s}$, -NH-), 12.29 (1H, s, OH). Anal. Calcd. for $\mathrm{C}_{17} \mathrm{H}_{18} \mathrm{~N}_{2} \mathrm{O}_{2} \mathrm{~S}$; C: \% 64.56; H: \% 5.66; N: \% 8.85; S: \% 9.83. Found: C: \% 64.94; H: \% 5.77; N: \% 8.91; S: \% 10.20 .

(4-\{[(Phenylcarbonyl)thiocarbamoyl $]$ amino $\}$ phenyl)acetic acid (11) UV $\lambda_{\max .}(\mathrm{EtOH})(\mathrm{nm})(\log \mathrm{e}): 266(4,18)$. IR $\left(\mathrm{u}_{\max } \mathrm{cm}^{-1}\right)$ : 3284 (N-H, O-H), 3000-2950 (C-H), 1666,1597 (C=O), 1263 (C=S). ${ }^{1} \mathrm{H}-\mathrm{NMR}(400 \mathrm{MHz})$ (DMSO-d $\left.6 / \mathrm{TMS}\right) \mathrm{d}(\mathrm{ppm}): 3.59$ $\left(2 \mathrm{H}, \mathrm{s},-\mathrm{CH}_{2}\right), 7.23-8.04(9 \mathrm{H}, \mathrm{m}$, aromatic protons $), 11.55(1 \mathrm{H}, \mathrm{s}$, -NH-), 12.23 (1H, s, -NH-), $12.58(1 \mathrm{H}, \mathrm{s}, \mathrm{OH})$. Anal. Calcd. for $\mathrm{C}_{16} \mathrm{H}_{14} \mathrm{~N}_{2} \mathrm{O}_{3} \mathrm{~S}$; C: \% 61.13; H: \% 4.49; N: \% 8.91; S: \% 10.20. Found: C: \% 61.01; H: \% 4.78; N: \% 8.34; S: \% 9.36.

\subsection{Pharmacology}

Male and female adult Balb/C mice weighing 20-30 g were used. The animals were housed in colongy cages, under standard laboratory conditions, with free access to food and tap water. Room temperature and relative humidity were maintained at $22 \pm 1{ }^{\circ} \mathrm{C}$ and $60 \%$ respectively. A $12 \mathrm{hr} / 12$ hour (8 a.m./8 p.m.) light-dark cycle was used. All testing was conducted in the light phase of the day. After the adaption period of 2 days, experimental groups were chosen randomly. Each mouse was used only once. The experimental protocols were approved by the Animal Care and Use Committee of Marmara University (16.04.2009-02.2009.mar).

\subsubsection{Anticonvulsant Activity}

The anticonvulsant activity of the new compounds was determined by using PTZ (Sigma) and MES tests. All synthesized compounds were suspended in $0.5 \%$ methyl cellulose and administered at the dose of $50 \mathrm{mg} / \mathrm{kg} 30$ minutes prior the tests. Effective dose $50\left(\mathrm{ED}_{50}\right)$ value for PTZ $(60 \mathrm{mg} / \mathrm{kg})$ and convulsive current $50\left(\mathrm{CC}_{50}\right)$ of animals and it's $95 \%$ fiducial limits were calculated by the method of Litchfield and Wilcoxon (21).

Statistical analysis were evaluated using one way analysis of variance (ANOVA) followed by unpaired Student's t-test using Prism 3.0 (GraphPad Software, San Diego; CA; USA).

\subsubsection{PTZ test}

The animals of the control group received same volume of saline and standart drug was carbamazepine in PTZ test. Thirty minutes after the administration of the test compounds, all mice were injected with PTZ $60 \mathrm{mg} / \mathrm{kg}$ intraperitoneally and observed for 15 minutes. Motor responses were graded 0-5 according to the scale of Racine where, grade 1: no movements, grade 2: head twitching and myoclonic jerks $(\mathrm{MKJ})$, grade 3 : clonic forelimb convulsions, grade 4 : three plus change in posture, grade 5: falling back and generalized convulsions with tonic extention (22).

\subsubsection{MES test}

MES test was performed 30 minutes after the administration of the test compounds. The electroshocks were evoked through a current transmitter producing square waves (Ar1 Techinical ECT unit). In the MES test, seizures were elicited with a $60-\mathrm{Hz}$ alternating current of $25 \mathrm{~mA}$ intensity in Balb/c mice. The cur- 
TABLE 3. Anticonvulsive properties of compound $1 \mathrm{~b}$ in PTZ test

\begin{tabular}{|c|c|c|c|c|c|c|c|}
\hline Parameter & Onset time & Grade I \% & Grade II \% & Grade III \% & Grade IV \% & Grade V \% & $\begin{array}{c}\text { Survival } \\
\%\end{array}$ \\
\hline PTZ & $1.20 \mathrm{sec}$ & 99 & 80 & 75 & 71 & 52 & 50 \\
\hline $1 b$ & $2.58 \mathrm{sec}$ & $64^{\star \star \star}$ & $42^{\star \star \star}$ & $35^{\star \star \star}$ & $21^{\star \star \star}$ & $10^{\star \star \star}$ & $95^{\star \star \star}$ \\
\hline
\end{tabular}

rent was applied via ear clip electrodes for $450 \mathrm{~ms}$. In order to apply the shock, electrodes were attached to each animal's ears and the animals lay on their backs, their tails being fixed. Thus observation of the tonic and clonic convulsions that appeared during the seizure was ensured (23).

\section{RESULTS AND DISCUSSION}

\subsection{Chemistry}

A series of new thiourea derivatives were prepared according to Figure (3). Target compounds 1a-11 were prepared by reacting of equimolar 4-(aminophenyl)acetic acid and various isocyanates in acetone. The new compounds were isolated in satisfactory yields (42-70\%) and purified by recrystallisation from acetonitril. The purity of the compounds checked by TLC and elemental analyses. Both analytical and spectral data of all the synthesized compounds were in full agreement with the proposed structures. Physical and chemical properties of all compounds are presented in Table (1).

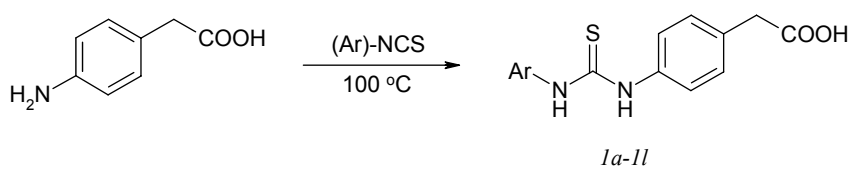

FIGURE 3. Synthesis scheme of compounds 1a-11

In general, IR spectra showed the $\mathrm{OH}$ and $\mathrm{NH}$ stretching vibrations at $3161-3564 \mathrm{~cm}^{-1}$, the $\mathrm{C}=\mathrm{O}$ stretching vibrations at $1666-1695 \mathrm{~cm}^{-1}$ and the $\mathrm{C}=\mathrm{S}$ stretching vibrations at $1224-1300$ $\mathrm{cm}^{-1}$. In the ${ }^{1} \mathrm{H}-\mathrm{NMR}$ spectrum, thiourea $\mathrm{NH}$ signals were determined at 9.55-12.23 ppm as two different singlets. The $\mathrm{OH}$ signals of carboxylic acide were observed at 11.98-12.58 ppm as singlet. The protons belonging to the aromatic ring and the other aliphatic groups are observed with the expected chemical shift and integral values. APCI-MS spectra of the selected compounds showed correct molecular ion peaks $\left(\mathrm{MH}^{+}\right)$which confirmed their molecular weights.

\subsection{Anticonvulsant Activity}

The anticonvulsant activity of the new compounds was determined by using PTZ (Sigma) and MES tests. The use of current animal models in the discovery of new AEDs development has advantages. The advantages include the use of intact rodents as easy models that detect anticonvulsant effects regardless of the mechanisms of action. MES and PTZ testing can be used in highthroughput screening, as shown by the National Institutes of Health Anticonvulsant Screening Program. Furthermore, these models can provide insight into pharmacokinetic-pharmacodynamic relations, which are of value for human studies (17).

All compounds were suspended in $0.5 \%$ methyl cellulose and administered intraperitoneally at the dose of $50 \mathrm{mg} / \mathrm{kg} 30$ minutes prior the tests. The anticonvulsant potential of these compounds was invastigated by both PTZ and MES models and shown in Table 2. Within the context of the MES model none of the compounds tested showed an anticonvulsant effect. The results from PTZ model basically simulate petit mal seizures. The introduction of chloro group at 4- position of phenyl ring in thiourea moiety (compound $\mathbf{1 b}$ ) resulted better activity than bearing 4-fluoro, 4-nitro, 4-methoxy, 4-methlsulfanyl, 4-trifluoromethyl and 4-trifluoromethoxy group of phenyl ring in PTZ test. The compound $\mathbf{1 b}$ reduced convulsions in all types of grades (from grade I to V), therefore it increased convulsive threshould. It also increased onset time from 1.20 to $2.58 \mathrm{sec}$. and survival \% from 50 to 95 (Table 3). Therefore, the compound $\mathbf{1 b}$ has a potantial to be an anticonvulsant drug for petit mal seizures.

Interestingly we expected the compound 1c which had chloro group at 2-, 4-, and 6-position of phenyl ring, displayed good activity because of lipophpilicity. But it was found less potent than compound $\mathbf{1 b}$ having one chloro goup at 4-position on phenyl ring. The thioureas bearing benzyl, phenylethyl or phenylcarbonyl were inactive both PTZ and MES test.

\section{Sübstitüe Tiyoüre Türevlerinin Sentezi ve Antikonvulsan Aktiviteleri}

ÖZET: 4-Aminofenilasetik asitin sübstitüe izotiyosiyanatlar ile reaksiyonu sonucu, on iki adet yeni tiyoüre bileşiği sentezlenmiştir. Bileşiklerin kimyasal yapıları IR, 1H-NMR, kütle spektroskopisi ve elementel analiz testleri ile aydınlatılmıştır. Tüm bileşiklerin antikonvulsan aktiviteleri $50 \mathrm{mg} / \mathrm{kg}$ dozda farelerde pentilentetrazol (PTZ) ve maksimal elektroşok nöbet (MES) testleri kullanılarak tayin edilmiştir. Bileşik 1b'nin (4-\{[(4-klorofenil)tiyokarbamoil]amino\}fenil)asetik asit, diğer bileşiklere oranla daha aktif olduğu saptanmıştır. Tüm seviyelerde konvulsiyon oranını düşüren 1b bileşiği aynı zamanda nöbet eşiğini de yükseltmiştir. Ayrıca nöbet başlangıç süresini 1.20 saniyeden 2.58 saniyeye, hayatta kalma oranını ise $\% 50$ 'den $\% 95$ 'e yükseltmiştir.

ANAHTAR KELIMELER: Sentez, tiyoüre, antikonvulsan aktivite. 
In conclusion, a series of thiourea derivatives have been synthesized and screened for their anticonvulsant activity. The anticonvulsant screening indicated that among the tested compounds, thiourea derivative carrying $4-\mathrm{Cl}$ group on the phenyl ring exhibited noteworthy activity in PTZ test. From these data, ideas for future molecular modification leading to compound with greater favorable pharmacological properties may be derived.

\section{ACKNOWLEDGEMENT}

The authors wish to thank Marmara University Scientific Research Projects Commission (BAPKO, Project number, SAG-CYLP-270109-0013, 2009) to financial support for this study.

\section{REFERENCES}

1. Bell FW, Cantrell AS, Högberg M, Jaskunas SR, Johansson NG, Jordan CL, Kinnick MD, Lind P, Morin JM, Noreen R, Öberg B, Palkowitz JA, Parrish CA, Pranc P, Sahlberg C, Ternansky RJ, Vasileff RT, Vrong L, West SJ, Zhang H, Zhou XX. Phenethylthiazolethiourea (PETT) compounds, a new class of HIV-1 reverse transcriptase inhibitors. 1.Synthesis and basic structure-activity relationship studies of PETT analogs. J Med Chem 1995; 38(25): 4929-4936.

2. Uçkun FM, Pendergrass $S$, Maher $D$, Zhu D, Tuel-Ahlgren L, Mao C, Venkatachalam TK. N'-[2-(2-thiophene) ethyl]-N'-[2-(5-bromopyridyl)] thio-urea as a potent inhibitor of NNI-resistant and multidrug-resistant HIV-1. Bioorg Med Chem Lett 1999; 9(24): 3411-3416.

3. Küçükgüzel İ, Tatar E, Küçükgüzel ŞG, Rollas $S$, De Clercq E. Synthesis of some novel thiourea derivatives obtained from 5-[(4-aminophenoxy)methyl]-4-alkyl/ aryl-2,4-dihydro-3H-1,2,4-triazole-3-thiones and evaluation as antiviral/ anti-HIV and anti-tuberculosis agents. Eur J Med Chem 2008; 43(2):381-392.

4. Todoulou OG, Papadaki-Valiraki AE, Filippatos EC, Ikeda, S, De Clercq E. Synthesis and anti-myxovirus activity of some novel N,N'-disubstituted thioureas. Eur J Med Chem 1994; 29(2): 127-131.

5. Sriram D, Yogeeswari $P$, Madhu K. Synthesis and in vitro antitubercular activity of some 1-[(4-sub)phenyl]3-(4-\{1-[(pyridine-4-carbonyl) hydrazono] ethyl $\}$ phenyl) thiourea. Bioorg Med Chem Lett 2006; 16: 876-878.

6. Dixit PP, Patil VJ, Nair PS, Jain S, Sinha N, Arora SK. Synthesis of 1-[3-(4-benzotriazol-1/2-yl-3-fluorophenyl)2-oxo-oxazolidin-5-ylmethyl]-3-substituted-thiourea derivatives as antituberculosis agents. Eur J Med Chem 2006; 41: 423-428.

7. Küçükgüzel İ, Küçükgüzel ŞG, Rollas S, Kiraz M. Some 3-thioxo/alkylthio-1,2,4-triazoles with a substituted thiourea moiety as possible antimycobacterials. Bioorg Med Chem Lett 2001; 11: 1703-1707.

8. Saeed A, Shaheen U, Hameed A, Haider Naqvi SZ. Synthesis, characterization and antimicrobial activity of some new 1-(fluorobenzoyl)-3-(fluorophenyl) thioureas. J of Fluor Chem 2009; 130(11): 1028-1034.

9. Chung J, Kim SY, Lim J, Choi H, Kang S, Yoon H, Ryu H, Kang DW, Lee J, Kang B, Choi S, Toth A, Pearce LV, Pavlyukovets VA, Lundberg DJ, Blumberg PM. a-Substituted N-(4-tert-butylbenzyl)-N'-[4-(methylsulfonylamino)- benzyl]thiourea analogues as potent and stereospecific TRPV1 antagonists. Bioorg Med Chem 2007; 15: 60436053.

10. Dos Santos L, Lima LA, Cechinel-Filho V, Corrêa R, Buzzi FC, Nunes RJ. Synthesis of new 1-phenyl-3-\{4-[(2E)-3phenylprop-2-enoyl]phenyl\}-thiourea and urea derivatives with anti-nociceptive activity. Bioorg Med Chem 2008; 16: 8526-8534.

11. Mahajan A, Yeh $S$, Nell M, Van Rensburg CJ, Chibale K. Synthesis of new 7-chloroquinolinyl thioureas and their biological investigation as potential antimalarial and anticancer agents. Bioorg Med Chem Lett 2007; 17: 56835685.

12. Karakuş $S$, Küçükgüzel ŞG, Küçükgüzel İ, De Clercq $E$, Pannecouque C, Andrei G, Snoeck R, Şahin F, Bayrak ÖF. Synthesis, antiviral and anticancer activity of some novel thioureas derived from N-(4-nitro-2-phenoxyphenyl)methanesulfonamide. Eur J Med Chem 2009; 44(9): 35913595.

13. Liu J, Song B, Fan H, Bhadury PS, Wan $W$, Yang $S, X u$ $\mathrm{W}, \mathrm{Wu} \mathrm{J}$, Jin L, Wei X, Hu D, Zeng S. Synthesis and in vitro study of pseudo-peptide thioureas containing a-aminophosphonate moiety as potential antitumor agents. Eur J Med Chem 2010; 45(11): 5108-5112.

14. Heinisch G, Matuszczak B, Rakowitz D, Tantisina B. Synthesis of $\mathrm{N}$-aryl-N'-heteroaryl-substituted urea and thiourea derivatives and evaluation of their anticonvulsant activity. Arch Pharm (Weinheim) 1997; 330(7): 207210.

15. Masereel B, Lambert DM, Dogne JM, Poupaert JH, Delarge J. Anticonvulsant activity of 3-pyrid-3-yl-sulfonyl ureas and thioureas. Epilepsia 1997; 38(3): 334-337.

16. Pandeya SN, Manjula H, Stables JP. Design of semicarbazones and their bioisosteric analoques as potential anticonvulsants. Pharmazie 2001; 56(2): 121-124.

17. Perucca E, French J, Bialer M. Development of new antiepileptic drugs: challenges, incentives, and recent advances. Lancet Neurol 2007; 6: 793-804.

18. Koçyiğit-Kaymakçıŏlu B, Rollas S, Korcegez E, Aricioglu F. Synthesis and biological evaluation of new N-substituted-N'-(3,5-di/1,3,5-trimethylpyrazole-4-yl) thiourea/urea derivatives. Eur J Pharm Sci 2005; 26(1): 97-103.

19. Karakuş S, Koçyiğit-Kaymakçıŏlu B, Toklu HZ, Arıcıoğlu F, Rollas S. Synthesis And Anticonvulsant Activity of New N-(Alkyl/Substitutedaryl) - N'-[4(5-Cyclohexylamino)-1,3,4-Thiadiazole-2-yl) Phenyl] Thioureas. Archive der Pharmazie 2009; 342(1): 48-53.

20. Pandeya SN, Mishra V, Sign PN, Rupainwar DC. Anticonvulsant activity of thioureido derivatives of acetophenone semicarbazone. Pharmacol Res 1999; 37(1): 17-22.

21. Litchfield JT, Wilcoxon FA. A simplified method of evaluating dose-effect experiments. J Pharmacol Exp Ther 1949; 96: 99-113.

22. Racine RJ. Modification of seizure activity by electrical stimulation. II. Motor seizure. Electroencephalogr Clin Neurophysiol 1972; 32: 281-294.

23. Krall RL, Penry JK, White BG, Kupferberg HJ, Swinyard EA. Anticonvulsant drug development: Anticonvulsant drug screening. Epilepsia 1978; 19: 409-428. 